\title{
Artificial intelligence in underground development: a study of TBM performance
}

\author{
A. Benardos \\ National Technical University of Athens, \\ School of Mining \& Metallurgical Engineering, Greece
}

\begin{abstract}
Modelling tunnel boring machine (TBM) performance is an important aspect in tunnel operations. The use of artificial intelligence techniques such as artificial neural networks has been recently introduced to this subject and the results from such applications prove their potential in making accurate prognosis. This paper presents a review of feed-forward artificial neural network (ANN) development and furthermore it illustrates their application by the use of two cases studies from Italian and Greek underground projects, where the TBM performance is modelled. The results obtained show that the developed ANNs can efficiently generalise the TBM behaviour in their respective geotechnical environment, having a reliable, effective and consistent performance.
\end{abstract}

Keywords: TBM performance modelling, artificial neural networks.

\section{Introduction}

Assessing the performance of tunnelling operations is one of key data for the overall success of the project as this issue is directly interconnected with the financial performance of the construction works [1]. Even more important is to estimate the tunnelling rate of tunnel boring machines (TBMs), as the flexibility limitations these particular machines have can lead to considerable downtime. These problems are more intense in tunnelling projects constructed in complex geological formations [2] and especially in urban areas where the low construction depth and the external loading from the buildings increase risk conditions [3].

In order to assess the performance of TBMs many researchers have proposed various methodologies [4-12] in an effort to express the penetration rate using as 
inputs data relating to the rock mass properties and/or machine characteristics. Beyond mathematical formulae and analytical solutions, artificial intelligence systems and more particularly artificial neural networks (ANNs) have not been introduced in this issue until recently. Nevertheless, many researchers [13-17] have demonstrated very promising results. This is because ANNs can further enhance the effectiveness of the analysis, especially in rock engineering applications such as the one described, where the interrelated parameters are numerous; their interaction is not clearly identified, or is very complicated to be explicitly expressed.

This paper gives a brief review regarding ANN development and furthermore it deals with the modelling of the TBM performance emphasising the identification of the performance oscillations throughout the tunnelling period. This is made possible by the development of ANNs capable of learning from the tunnelling experience and generalising solutions for new sets of input data. Hence, the main aim is to produce a tailor-made model, utilised during the construction period, capable of providing estimates of the expected tunnelling advance rate. To illustrate the efficiency and accuracy of the ANN generalisation two case studies are presented in Italian and Greek underground projects, where the TBM penetration rate is modelled with respect to the geological and geotechnical conditions, as well as the machine characteristics by the use of trained neural networks.

\section{Artificial neural networks}

\subsection{Definition}

The development of ANNs started as an attempt to understand the operation of the human brain and mimic its assessment capabilities. In other words, to be able to decide and act under uncertainty or even deal with situations having limited previous experience. ANNs are mathematical models consisting of interconnected processing nodes (neurons) under a pre-specified topology (layers).

Neural networks have a strong similarity to the biological brain and therefore a great deal of their terminology is borrowed from neuroscience. Their basic characteristic is the ability to perform massively parallel computing of the input stimulus (data), contrary to the custom mathematical models that are based rather on a serial process of mathematical and logical functions [18]. Another advantage of the ANNs is their flexibility in data processing, as no deterministic mathematical relationship of the examined components is required. Instead, once the data is introduced, in a cause-effect mode, the network identifies the existing relationships, learns and mimics their behaviour by adjusting the strength of the links between the neurons (connection weights). Thus, they cannot be programmed but they are rather taught through case experience. As a result, soon after the ANN's training, given an existing dataset, estimates can be drawn for another specific data input. Thus, the trained network can generalise and give estimates for uncertain conditions or even incomplete data [19]. The main disadvantage of ANNs is that an explicit determination of the parameter's 
weighting is not an easy task or it may not even be possible in large and complex network architectures. The ANN operation is based on the following:

- Data processing occurs in a number of simple processing units (neurons), which have signal inputs and outputs.

- The neurons' bonding is made through connection links, each one of them having a corresponding weight that multiplies the signal.

- Each neuron applies an activation function to the signal input to control the signal output.

\subsection{ANN architecture and training process}

In general, a typical ANN topology is consisted by a set of layers; the input layer, one or more hidden layers and the output layer, each one of them containing a certain number of neurons. Accordingly, each neuron is linked to neighbours with varying coefficients of connectivity that represent the weighting of these connections. Each neuron of the hidden layer(s) is interconnected to all others found in the input and output layers.

The type of ANN used in this paper are the feed-forward neural networks, which are the most widely used. They are commonly applied to problems where a set of input vectors should be corresponded to another specified set of output vectors. The training procedure consists of a sequential data feed into the network, followed by the comparative evaluation of the corresponding output provided by the ANN and the actual result. The network adjusts the weighting of the connection links in the neurons of the hidden layers in a continuous effort to produce the results that would best correspond to the training dataset. A complete pass of all the input data through the network consists a training epoch and usually a great number of epochs is required for the residual error to converge below a pre-specified threshold. A schematic illustration of a feedforward ANN training is given in fig. 1.

Feed-forward ANNs are usually trained with the backpropagation algorithm, also known as the generalized delta rule. In order to train a feed-forward ANN, corresponding sets of input (training input vectors) and output (target output

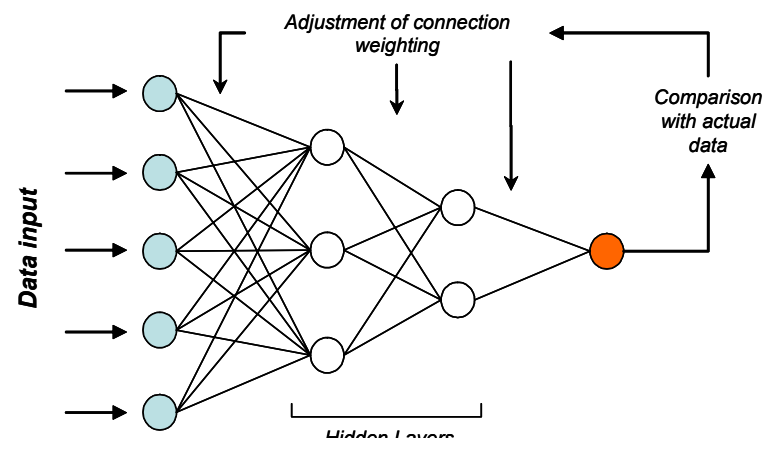

Figure 1: $\quad$ Training process of a feed-forward ANN with two hidden layers. 
vectors) data must be presented to it. Each target output vector is the ANN's desired response to the appropriate training input vector. The training algorithm is used to modify the connection weights so as to minimize the error between the ANN's output and its desired response for all training input vectors. Generally, the training error is a function of the difference between the ANN's predicted values and desired responses, with the connection weights as the independent variables. Common formulations used for the training error include the sum squared error (SSE) and the mean squared error (MSE).

\section{ANN in TBM performance prediction - cases studies}

The whole idea follows the ANN philosophy, that is, to analyse the experience gained from the tunnel boring process and to correspond it to a set of selected data. This cause-effect request is used in the ANN so as to identify the interactions between the data and to come up with the appropriate weighting of the parameters involved, which will finally determine the generalisation accuracy.

In order to illuminate the ability of ANNs to generalise solutions depicting the TBM performance, two case studies are presented. The first one deals with two Italian tunnels (Maen and Pieve), where the penetration rate is modelled based on input data relating to ground properties and machine characteristics. The second is related to an interstation tunnel, from the Athens metro project, where an ANN is trained based on a series of geotechnical data in order to be able to reveal possible risk prone areas where TBM operation is negatively affected by ground conditions. All cases are modelled individually, as the geotechnical environment, as well as, the particular characteristics of each TBM used are different. Thus, the development of separate ANNs enhances the precision and the efficiency of the generalisations that could be further used in order to have consistent prognosis for the corresponding geotechnical setting.

\subsection{Case study 1 - Italian tunnels}

Data for TBM performance analysis have been obtained from two tunnels (Maen, Pieve) excavated in metamorphic rocks located in the Italian Alps. The combined tunnel length is approximately $11.5 \mathrm{~km}$, while data records exist for the $8.5 \mathrm{~km}$. In the Maen tunnel the recordings were made at a $5 \mathrm{~m}$ interval, whereas at the Pieve tunnel data relating to the geotechnical conditions was gathered on a daily basis [10]. Details on the specific tunnel projects and the TBMs used are given in Table 1 , while in Table 2 data relating to the characteristics of the geological formations encountered is presented.

Regarding the lithological types (categorical target variables) that were introduced in the ANN, for each tunnel, each one of them has been corresponded to an input neuron using the "one-of-c" coding principle. That means that the coding of $\mathrm{c}$ binary target variables ( 0 or 1$)$ corresponds to the c categories. These new variables are also known as "dummy" variables and for each one the zero value is assigned to it, except for the one corresponding to the correct category, which is given the value one. Thus, only the neuron that corresponds to the 
Table 1: $\quad$ Construction data for the tunnels under investigation [10].

\begin{tabular}{|l|c|c|}
\hline & Maen & Pieve \\
\hline Surveyed section length $(\mathrm{m})$ & 1750 & 6400 \\
\hline Total excavation time (days) & 413 & 809 \\
\hline Excavated diameter $(\mathrm{m})$ & 4.20 & 4.05 \\
\hline Tunnel slope $\left({ }^{\circ}\right)$ & $24-35$ & $\approx 0$ \\
\hline TBM model & Wirth 340/420 E & Robbins 1111-234/3 \\
\hline TBM type & Open & Double shield \\
\hline Number of cutters & 36 & 27 \\
\hline Cutter diameter (in) & $177^{\prime}$ & 17 \\
\hline Maximum trust $(\mathrm{kN})$ & 7920 & 4602 \\
\hline Boring stroke (m) & 1.5 & 0.63 \\
\hline Cutterhead rotation rate (rpm) & $5.5-11$ & 11.3 \\
\hline
\end{tabular}

Table 2: $\quad$ Main characteristics of the geological formations [10].

\begin{tabular}{|c|c|c|c|c|c|c|c|}
\hline Tunnel & Rock type & $\begin{array}{c}\text { UCS } \\
(\mathrm{MPa})\end{array}$ & $\begin{array}{c}\text { Tensile } \\
\text { strength } \\
(\mathrm{MPa})\end{array}$ & $\begin{array}{l}\text { Mean } \\
\text { Mohs' } \\
\text { hardness }\end{array}$ & $\begin{array}{c}\text { Knoop } \\
\text { hardness } \\
(\mathrm{GPa})\end{array}$ & $\begin{array}{l}\text { Cutter } \\
\text { Life } \\
\text { Index }\end{array}$ & $\begin{array}{l}\text { Young's } \\
\text { modulus } \\
(\mathrm{GPa})\end{array}$ \\
\hline \multirow{5}{*}{$\frac{\tilde{E}}{\stackrel{E}{\Sigma}}$} & Serpentinite & 124 & - & 3.6 & - & $30-70$ & - \\
\hline & Metabasite & 180 & 15 & 6.2 & 6.2 & $10-20$ & 65 \\
\hline & Chlorite schist & 17 & - & 2.8 & - & $60-90$ & - \\
\hline & Metagabbro & 138 & $10-12$ & 6 & 5.1 & $15-25$ & 39 \\
\hline & Calc schist & 75 & - & 3.6 & - & $30-70$ & - \\
\hline \multirow{4}{*}{$\frac{0}{2}$} & Micaschist & $\begin{array}{c}124- \\
215\end{array}$ & $5-9$ & 4.1 & $5.2-8.5$ & $15-70$ & 28 \\
\hline & Metadiorite & $\begin{array}{c}171- \\
221\end{array}$ & $8-13$ & 5.1 & $6.2-7.0$ & $15-40$ & $\begin{array}{l}46- \\
100\end{array}$ \\
\hline & $\begin{array}{c}\text { Meta } \\
\text { quartzdiorite }\end{array}$ & $\begin{array}{l}160- \\
210 \\
\end{array}$ & - & 6.4 & - & 15 & - \\
\hline & Metagranite & $\begin{array}{c}146- \\
296\end{array}$ & $0.7-7$ & 6.6 & $7-10$ & 10 & $24-38$ \\
\hline
\end{tabular}

Table 3: "One-of-c" coding used for the lithologies in the Maen tunnel case.

\begin{tabular}{|c|c|c|c|c|c|c|c|c|}
\hline \multirow{6}{*}{$\frac{\tilde{\mathbb{E}}}{\sum^{\frac{\pi}{2}}}$} & SP & Serpentinite & 1 & 0 & 0 & 0 & 0 & 0 \\
\hline & CHLSC & Chlorite schist & 0 & 1 & 0 & 0 & 0 & 0 \\
\hline & TALC & Talc schist & 0 & 0 & 1 & 0 & 0 & 0 \\
\hline & CLS & Calc schist & 0 & 0 & 0 & 1 & 0 & 0 \\
\hline & MBAS & Metabasite & 0 & 0 & 0 & 0 & 1 & 0 \\
\hline & MG & Metagabbro & 0 & 0 & 0 & 0 & 0 & 1 \\
\hline
\end{tabular}

actual encountered lithological type, for the given data array, is activated each time. The "One-of-c" coding used for the lithologies in the Maen tunnel case is given in Table 3.According to the above, for the Maen tunnel there were 8 input neurons (6 for the lithological types), while for the Pieve tunnel the number of the input neurons were 7 ( 5 for the lithological types).

The datasets from these two tunnels have been discerned into 3 subsets using a uniform sampling process; the training, the testing and the validation ones. 
From the 330 datasets for the Maen case and the 301 datasets available in the Pieve tunnel, about $60 \%$ was used for training, whereas the testing and validation subsets each amounted approximately $20 \%$ of the data. The training dataset is introduced to the ANN so as to properly adjust the weighting connections of the neurons against target output (see fig. 1), while the validation subset is used as a barrier to avoid data overfitting, as it stops the training when designated error levels are reached. Finally, the testing subset is used as the measure of evaluating the trained model's efficiency. The input data of this subset are unknown to the model as they are used only after the completion of the training process. The comparison of the model's estimates with the actual output data, documents ANN's ability to generalize (predict). The ANN's performance is assessed in terms of the relative error level $(\Delta)$ achieved, between the actual $\left(P R_{\text {actual }}\right)$ and the predicted penetration rates $\left(P R_{\text {predicted }}\right)$, following the expression:

$$
\Delta=\frac{P R_{\text {actual }}-P R_{\text {predicted }}}{P R_{\text {actual }}}
$$

This criterion can provide a clear aspect regarding the ANN behaviour and moreover makes possible the comparison between the ANN results and other methods or theoretical models focusing on advance rate prediction.

In both cases, the optimal results were obtained by utilizing two hidden layers, with an increased number of neurons in the first of them. In Table 4, the optimum ANN architectures for the two tunnel cases are given, along with the mean squared errors (MSE) of the training process and the relative error levels $(\Delta)$ for the generalisation outputs. The most efficient behaviour is achieved in the ANN developed for the Maen tunnel, having an $8 \times 9 \times 5 \times 1$ architecture. This particular structure type means that the ANN has a total of 4 layers, with 8 neurons in the input level, same as the number of the parameters, two hidden layers with 9 and 5 neurons respectively, followed by 1 neuron in the output layer that eventually generates the value of the penetration rate.

Table 4: $\quad$ ANN training and testing error for each examined tunnel.

\begin{tabular}{|l|c|c|}
\hline & Maen & Pieve \\
\hline Optimum ANN architecture & $8 \times 9 \times 5 \times 1$ & $7 \times 6 \times 5 \times 1$ \\
\hline Training MSE & 0,119 & 0,086 \\
\hline Relative error of ANN generalisation (\%) & $17,9 \%$ & $21,5 \%$ \\
\hline
\end{tabular}

Beyond the presentation of the mean values for the relative error levels it is of equal importance to evaluate the overall behaviour of the trained networks. This will assure that the ability of the ANNs to provide reliable prognosis is spread throughout the dataset and not only focused in particular sections. This check can be made with the use of fig. 2, where the actual penetration rate for the Maen tunnel are presented in conjunction with the ANNs' output for all data incorporated in the testing subset, along with an additional bar-graph presenting the attained relative error. Furthermore, in fig. 3 the scatter plot between actual and modelled penetration rate is given.

All the above concur that the ANNs' generalisations present a satisfactory approximation level, consistent throughout the dataset examined, and 

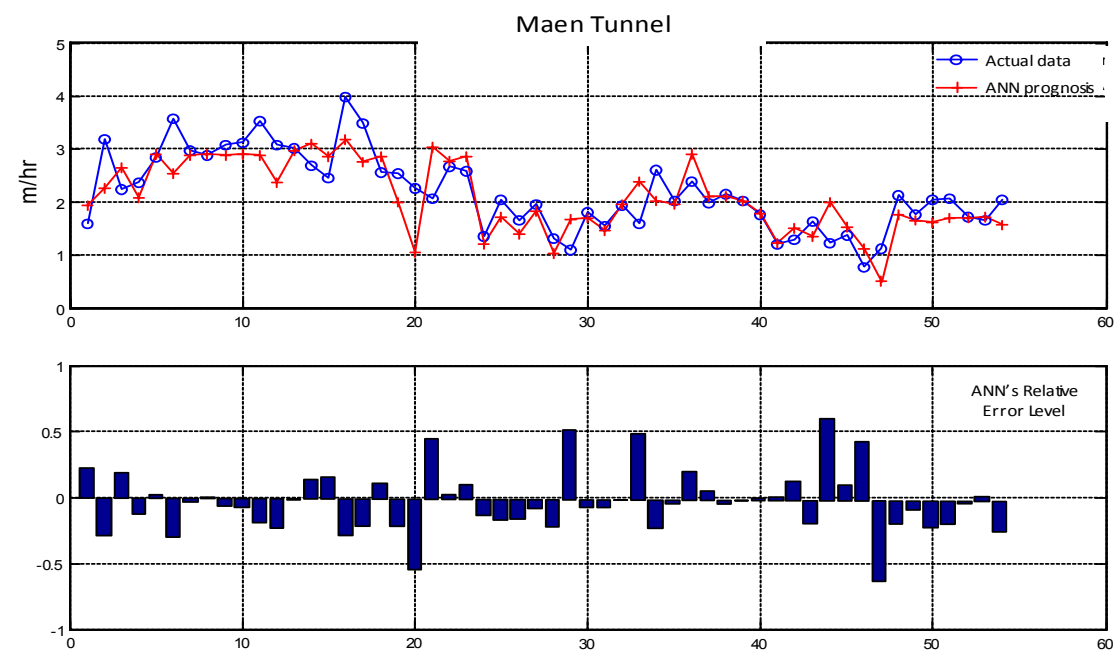

Figure 2: ANN generalisation for the complete testing subset of the Maen tunnel.

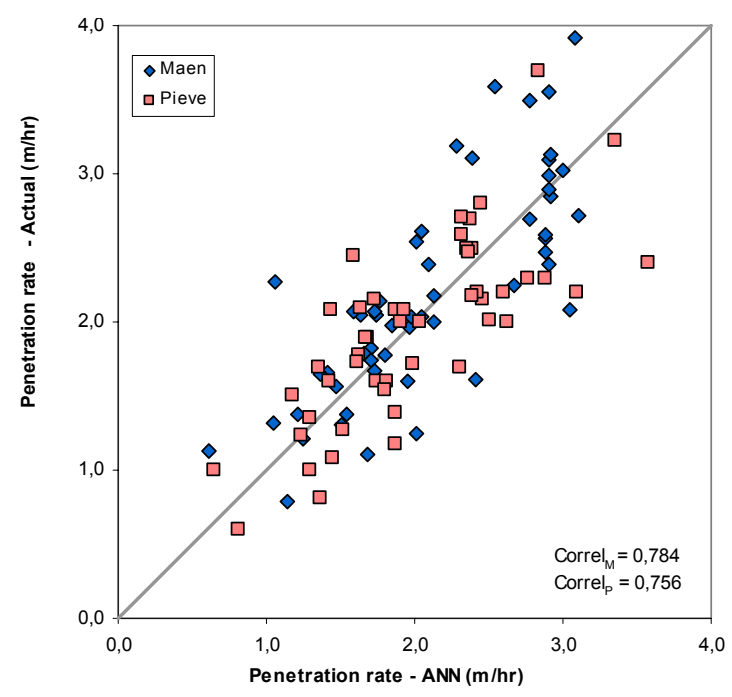

Figure 3: Scatter-plot of the measured PR values against the ANNs' predictions for the Maen and Pieve tunnels.

consequently through their respective tunnel sections. They follow the changes experienced in the actual TBM's penetration rate with satisfactory levels of accuracy and finally attain a correlation coefficient that exceeds $75 \%$ in the two examined case studies. 


\subsection{Case study 2 - Athens metro tunnel}

The examined tunnel is located between the Katehaki and Panormou stations. The geological setting is a system of low-level metamorphic sedimentary weak rock consisted of interbedded marly limestones, calcareous sandstones, siltstones, conglomerates, phyllites and schists. The formations are intensely thrusted, folded and faulted with a variable and erratic degree of weathering and alteration. This particular excavation is the longest interstation tunnel in the Athens Metro, until now, having a total length of $1129.36 \mathrm{~m}$ [20]. The surveyed tunnel length is approximately $1077 \mathrm{~m}$, after the exclusion of the first $53 \mathrm{~m}$ (learning curve period). The area is divided in 11 control areas (segments), in which, data from 16 boreholes is collected and the assessment of the selected geological properties is made. All data have been spatially modelled so as to identify the properties especially within the $12 \mathrm{~m}$ thick stratum that the tunnel is actually being built in, ranging, along the chainage, from the level of $+120 \mathrm{~m}$ to the level of $+156 \mathrm{~m}$. The ANN's inputs are based on data relating to the geological and geotechnical characteristics of the subsurface and the specific site conditions. Although machine characteristics (e.g. thrust, torque) are very important for the overall TBM performance, in the case where tunnelling is performed in soft rock or complex ground formations, the properties of the ground medium tend to be the most influential ones, as they govern the type and extend of possible failures. Subsequently, encountering ground conditions different from the TBM's working envelope, affect the achieved tunnelling rate [21] and can give rise to claims. Thus, the model considers the geological setting to be the most dominant factor for the TBM performance, as many researchers have also noted $[22,8,10]$, and all possible problems and downtime are a direct effect of the geotechnical conditions.

The selection of the parameters used in the model was made having in mind their capability to credibly represent the ground behaviour, hydrogeological environment and site-specific conditions [23]. These parameters are easily collected in the site-investigation phase and are available to all design stages of the project, without the need for implementing special investigation techniques. More specifically, these are:

- Rock mass fracture degree as represented by RQD

- Weathering degree of the rock mass

- Overload factor - stability factor (N)

- Rock mass quality represented by RMR classification

- Uniaxial compressive strength of the rock

- Overburden - construction depth

- Hydrogeological conditions represented by the water-table surface relative to the tunnel depth

- Rockmass permeability

For each segment, a corresponding value for every principal parameter is taken. Allocating a representative value for the parameters is accomplished by the spatial modelling of the parameter's value and by the incorporation of 
statistical distribution that characterise the parameter's behaviour in each segment [30].

In the next step, the data is categorised in 4 interval scale classes, from 0 to 3 , where 0 denotes the worst case and 3 the best. The limits taken in every class are representative of the specific site conditions and the machine characteristics. In the case of the Athens metro, the tunnel is constructed in relative low depth and, in general, in weak rock conditions with a double shield TBM machine. The rating of each parameter is presented in Table 5 .

Table 5: $\quad$ Rating of the parameters.

\begin{tabular}{|c|c|}
\hline \multicolumn{2}{|c|}{ Rockmass Fracture degree - RQD } \\
\hline Value Class & Rating \\
\hline$<10$ & 0 \\
\hline $10-30$ & 1 \\
\hline $30-60$ & 2 \\
\hline$>60$ & 3 \\
\hline
\end{tabular}

\begin{tabular}{|c|c|}
\hline \multicolumn{2}{|l|}{ Rockmass Weathering } \\
\hline Value Class & Rating \\
\hline Compl. Weath.-CW & 0 \\
\hline High Weath.-HW & 1 \\
\hline Med. Weath.-MW & 2 \\
\hline SW, Fresh & 3 \\
\hline
\end{tabular}

\begin{tabular}{|c|c|}
\hline Overload Factor $(\mathrm{N})$ & Rating \\
\hline Value Class & 0 \\
\hline$>5$ & 1 \\
\hline $3-5$ & 2 \\
\hline $1,25-3$ & 3 \\
\hline$<1,25$ &
\end{tabular}

\begin{tabular}{|c|c|}
\hline \multicolumn{2}{|c|}{ Rock Mass Rating - RMR } \\
\hline Value Class & Rating \\
\hline$<10$ & 0 \\
\hline $10-30$ & 1 \\
\hline $30-60$ & 2 \\
\hline$>60$ & 3 \\
\hline
\end{tabular}

\begin{tabular}{|c|c|}
\hline \multicolumn{2}{|l|}{ UCS (MPa) } \\
\hline Value Class & 0 \\
\hline$<2$ & 1 \\
\hline $2-15$ & 2 \\
\hline $15-40$ & 3 \\
\hline$>40$ &
\end{tabular}

\begin{tabular}{|c|c|}
\hline Water Table Surface (m) \\
\hline Value Class & Rating \\
\hline$>10$ & 0 \\
\hline $5-10$ & 1 \\
\hline $0-5$ & 2 \\
\hline$<0$ & 3 \\
\hline
\end{tabular}

\begin{tabular}{|c|c|}
\hline Overburden $(\mathrm{m})$ & Rating \\
\hline Value Class & 0 \\
\hline$<7,5$ & 1 \\
\hline $7,5-12,5$ & 2 \\
\hline $12,5-17,5$ & 3 \\
\hline$>17,5$ &
\end{tabular}

\begin{tabular}{|c|c|}
\hline Permeability (m/sec) & \\
\hline Value Class & Rating \\
\hline$<10^{-4}$ & 0 \\
\hline $10^{-4}-10^{-6}$ & 1 \\
\hline $10^{-6}-10^{-8}$ & 2 \\
\hline$>10^{-8}$ & 3 \\
\hline
\end{tabular}

The limits of the proposed rating transforms the continuous data to a discrete probability structure, a form that is finally used as input to the model. More specifically, the data is introduced to the ANN as the expected values (EV) of the parameters (Table 6). For example, given $V_{l}, V_{2}, \ldots, V_{n}$ values having a respective probability of occurrence $P_{1}, P_{2}, \ldots, P_{n}$, the expected value of the variable $X$, is estimated as:

$$
E[X]=E V=\sum_{i=1}^{n} P_{i} \cdot V_{i}, \quad \text { while, } \sum_{i=1}^{n} P_{i}=1
$$

The tunnelling advance rate (AR), recorded in each segment (Table 7), is also introduced into the ANN model. Hence, the input vector of the parameters is tallied to the output vector of the mean achieved advance rate, in each segment, expressed in $\mathrm{m} /$ day [21]. Note that all externally originated delays (e.g. strikes, maintenance, etc.) have not been taken into account. 
Table 6: $\quad$ Expected values of the parameters in each segment.

\begin{tabular}{|l|c|c|c|c|c|c|c|c|c|c|c|}
\hline Parameter & Seg1 & Seg2 & Seg3 & Seg4 & Seg5 & Seg6 & Seg7 & Seg8 & Seg9 & Seg10 & Seg11 \\
\hline RQD & 0.13 & 0.88 & 0.90 & 0.64 & 0.72 & 1.37 & 1.62 & 1.26 & 0.55 & 0.66 & 0.74 \\
\hline $\begin{array}{l}\text { Rockmass } \\
\text { Weathering }\end{array}$ & 2.52 & 2.52 & 2.24 & 1.97 & 1.99 & 1.89 & 1.95 & 1.93 & 1.96 & 1.94 & 1.93 \\
\hline Overload Factor & 1.07 & 0.89 & 1.92 & 1.99 & 2.73 & 2.16 & 2.49 & 2.28 & 2.43 & 2.61 & 2.43 \\
\hline Rock Mass Rating & 0.00 & 0.00 & 0.36 & 0.93 & 1.10 & 1.83 & 2.00 & 1.49 & 1.08 & 1.00 & 1.00 \\
\hline UCS & 0.48 & 0.57 & 0.97 & 1.06 & 1.68 & 1.31 & 1.28 & 1.20 & 1.16 & 1.21 & 1.15 \\
\hline Overburden & 0.42 & 1.00 & 1.17 & 1.97 & 2.86 & 2.35 & 1.16 & 1.45 & 1.13 & 0.99 & 0.88 \\
\hline $\begin{array}{l}\text { Water Table } \\
\text { Surface }\end{array}$ & 3.00 & 2.32 & 1.71 & 1.00 & 0.23 & 0.02 & 0.94 & 1.40 & 2.17 & 2.40 & 2.75 \\
\hline Permeability & 1.92 & 1.97 & 1.95 & 1.89 & 1.86 & 1.69 & 1.90 & 1.82 & 1.86 & 1.76 & 1.81 \\
\hline
\end{tabular}

Table 7: $\quad$ Tunnelling advance rate data in each one of the control segments.

\begin{tabular}{|c|c|c|c|}
\hline Segment & Average AR (m/day) & Max AR (m/day) & Min AR (m/day) \\
\hline 1 & 4.00 & 8.8 & 0.0 \\
\hline 2 & 4.54 & 8.8 & 0.0 \\
\hline 3 & 6.25 & 10.4 & 2.8 \\
\hline 4 & 4.35 & 13.5 & 0.0 \\
\hline 5 & 9.82 & 12.1 & 0.5 \\
\hline 6 & 9.09 & 13.7 & 7.3 \\
\hline 7 & 16.67 & 21.0 & 14.7 \\
\hline 8 & 11.11 & 18.3 & 4.4 \\
\hline 9 & 10.85 & 17.0 & 6.1 \\
\hline 10 & 12.50 & 17.3 & 1.6 \\
\hline 11 & 14.07 & 14.8 & 10.4 \\
\hline
\end{tabular}

The dataset of the whole 11 segments has been divided into two subsets. The first one (training subset - $A$ ) is used for the ANN's training, whereas the second (testing subset - B) is used for assessing the model's generalisation capability. In order to ensure the ANN's performance the testing subset is consisted by the most representative segments, in terms of the achieved advance rate, namely segments no. 2, 7 and 9, as they represent the worst, the best and an average case. From the various network architectures that were examined, the ANN that was finally selected has an $8 \times 9 \times 4 \times 1$ topology. The mean squared error (MSE) of training approximates at $1.4 \times 10^{-27}$ and is attained after 103 training epochs. The results generated from the trained model were very satisfactory, as the elative error $(\Delta)$ between the model outputs and the testing subset ranges in the region of $6 \%$ and $8 \%$ (Table 8$)$.

Table 8: ANN generalisation output and actual AR data for the testing subset.

\begin{tabular}{|c|c|c|c|}
\hline Segment & ANN generalisation results & Actual data & Relative error \\
\hline 2 & 4.854 & 4.54 & 0.0693 \\
\hline 7 & 17.687 & 16.67 & 0.0610 \\
\hline 9 & 9.942 & 10.85 & -0.0837 \\
\hline
\end{tabular}




\section{Concluding remarks}

The utilization of artificial intelligence techniques, like the artificial neural networks, in TBM performance prediction can produce reliable solutions and can contribute in the efforts of their better understanding. This has been the case in the projects analysed in the paper, where the developed networks could efficiently and consistently generalise the behaviour of the three TBMs in their respective geotechnical environment.

The final remarks can be drawn:

- The use of ANN can provide an easy and user friendly modelling environment with enhanced capabilities.

- Once trained, the ANN can become an efficient tool for the prediction of the TBM's performance. It is a very flexible system and its feed with updated construction data could improve its accuracy and expand its applicability limitations.

- In terms of identification risk prone areas the use of investigation data in the ANN model could facilitate in the planning phase of tunnels, in selecting tunnel alignment, to the selection of TBM characteristics or even in selecting the most appropriate ground improvement technique.

As a final point, it should be noted that data and case records from projects already constructed could be gathered in a extensive database covering all aspects of physical, geological, geotechnical, as well as TBM and site specific characteristics. This could be an important first step to have a "universal" ANN development, which could integrate all past experience so as to generalise solutions and provide answers to all critical issues.

\section{Acknowledgements}

The author would like to thank Dr. M. Berti from the University of Bologna for providing the complete data of the Maen and Pieve tunnels and for giving his permission for their analysis in the context of this publication.

\section{References}

[1] Alber, M., Advance rates of hard rock TBM's and their Effect on Project Economics. Tunnelling and Underground Space Technology, 15(1), pp. 5564, 2000.

[2] Barla, G., \& Pelizza, S., TBM Tunnelling in difficult ground conditions. GeoEng 2000, Melbourne, 2000.

[3] Eisenstein, Z., Urban tunnelling challenges and progress. ITA 25th Anniversary Commemorative Book, 1999.

[4] Tarkoy, P.J., Predicting TBM penetration rates in selected rock types. Proc. Ninth Canadian Rock Mechanics Symposium, Montreal, 1973.

[5] McFeat-Smith, I., \& Tarkoy, P.J., Assessment of Tunnel Boring Performance. Tunnels and Tunnelling, pp. 33-37, 1979. 
[6] Bruland, A., Prediction model for performance and costs. Norwegian TBM Tunnelling, Norwegian Tunnelling Society, pp. 29-34, 1999.

[7] Sharp, W., \& Ozdemir, L., Computer modelling for TBM performance prediction and optimization. Proceedings, Int. Symp. on Mine Mechanization and Automation, CSM/USBM, pp. 57-66, 1991.

[8] Nelson, P.P., TBM performance analysis with reference to rock properties. Comprehensive rock engineering. Pergamon Press, pp. 261-291, 1993.

[9] Barton, N. TBM Tunnelling in jointed and faulted rock, Balkema, Rotterdam, pp.173, 2000.

[10] Sapigni, M., Berti, M., Bethaz, E., Busillo, A., \& Cardone, G., TBM Performance Estimation Using Rock Mass Classifications. Int. J. Rock Mech \& Min Sc., 39(6), pp. 771-788, 2002.

[11] Gong, Q.M., \& Zhao. J., Influence of rock brittleness on TBM penetration rate in Singapore granite. Tunnelling and Underground Space Technology, 22(3), pp.317-324, 2007.

[12] Yagiz, S., Utilizing rock mass properties for predicting TBM performance in hard rock condition. Tunnelling and Underground Space Technology, 23(3), pp. 326-339, 2008.

[13] Bruines, P., Neuro-fuzzy modelling of TBM performance with emphasis on the penetration rate. Memoirs of the Centre of Engineering Geology. Delft, no $173,1988$.

[14] Alvarez Grima, M., Bruines, P.A., \& Verhoef, P.N.W, Modelling tunnel boring machine performance by neuro-fuzzy methods. Tunnelling and Underground Space Technology, 15(3), pp. 259-269, 2000.

[15] Okubo, S., KFukui, K., \& Chen, W., Expert system for applicability of tunnel boring machines in Japan. Rock Mech. \& Rock Eng., 36(4), pp. 305322, 2003.

[16] Benardos, A.G., \& Kaliampakos, D.C., Modelling TBM performance with artificial neural networks. Tunnelling and Underground Space Technology, 19(6), pp. 597-605, 2004.

[17] Zhao, Z., Gong Q., Zhang Y., \& Zhao J., Prediction model of tunnel boring machine performance by ensemble neural networks. Geomechanics and Geoengineering, 2(2), pp. 123-128, 2007.

[18] Fausett, L., Fundamentals of neural networks. Architectures, Algorithms and Applications, Prentice Hall International Editions, 1994.

[19] Sietsma, J., \& Dow, J.F., Creating artificial neural networks that generalize. Neural Networks, 4, pp. 67-79, 1991.

[20] Attiko Metro SA. Interstation Katehaki-Panormou: General construction report, Attiko Metro, Athens, 1995.

[21] Deere, D.U., Adverse geology and TBM tunnelling problems. Proc. RETS, Society of Mining Engineers, vol. 1, pp. 574-586, 1981.

[22] Tarkoy, P.J., Tunnel boring machine performance as a function of local geology. Bul. Assoc. Engineering Geology, vol. xvii, no.2, pp. 41-44, 1981.

[23] Benardos, A.G. \& Kaliampakos, D.C., A methodology for assessing geotechnical hazards for TBM tunnelling - illustrated by the Athens Metro. Greece, Int. J. Rock Mech \& Min Sc., 41(6), pp. 987-999, 2004. 